\title{
Three-dimensional limit analysis of ancient masonry buildings with rigid block models
}

\author{
A. Orduña \\ Faculty of Civil Engineering, University of Colima, Mexico
}

\begin{abstract}
Limit analysis with rigid block models is a tool successfully used in recent years for the assessment of ancient masonry structural elements and small buildings. In this paper, the interface yield functions for three-dimensional models are defined at interpolation points, instead of using the generalised stresses approach. This approach leads to very simple expressions for the yield functions and flow rules and therefore, renders the mathematical programming problem easier to solve than the generalised stresses formulation. The solution for the limit analysis problem is obtained using the load-path following approach. The validation of the present proposal shows good agreement compared with non-linear finite element results. Keywords: limit analysis, rigid block assemblages, non-associated flow, numerical integration.
\end{abstract}

\section{Introduction}

A valuable model for the structural assessment of ancient masonry structures is the limit analysis of rigid block assemblages interacting through no-tension and frictional interfaces. The reason for this affirmation lies in the fact that masonry has low tensile strength and quasi-brittle failure; therefore, at collapse, the cracks render the structure as a set of rigid blocks rocking and sliding between them.

In the rigid blocks modelling strategy, the degrees of freedom are related to the blocks, and the stress and strain variables are related to the interfaces. Therefore, blocks can be regarded as extended nodes and the interfaces as structural elements. Previous works in this subject have used mostly a generalised stress approach for the interfaces [1-4]. This means that the generalised stresses are, for instance, the normal and shear forces and the bending and torsion moments. Besides, Livesley[5, 6], using a different approach, verified the contact at points located at the interface corners. 
In this paper, the use of numerical integration techniques is investigated, as an alternative to the generalised stresses approach for the yield function description. Firstly, the formulation for non-associated limit analysis of rigid block assemblages is outlined. Then, the formulation of the yield function and flow rule using numerical integration is presented. A discussion of the torsion failure on frictional interfaces follows, where it is emphasised the importance of a correct normal stress distribution calculation over the interface. A comparison of results for a model obtained by the proposed approach against non-linear FE method results serves to validate the proposal. Finally, relevant conclusions are stated.

\section{Limit analysis of rigid block assemblages}

Eqns. (1-6) are the conditions that a limit analysis solution with non-associated flow rule must fulfil, see e.g. [7]. Eqn. (1) combines the compatibility and flow rule conditions. Here, the columns of the matrix $\vec{N}_{0}$ contain the flow directions for each one of the yield functions in the structure; the flow multipliers for each one of such yield functions form the $\delta \vec{\lambda}$ vector; $\vec{C}$ is the compatibility matrix and $\delta \vec{u}$ is the vector of block displacement rates. Eqn. (2) is a scaling condition for the displacement rates that ensures the existence of non-zero but finite values. Here $\vec{F}_{v}$ is the vector of variable loads. Eqn. (3) ensures equilibrium. Here $\vec{F}_{c}$ is the vector of constant loads; $\alpha$ is the load factor that measures the amount of the variable loads applied to the structure and $\vec{Q}$ is the vector of generalised stresses at the interfaces. In this paper the elements of $\vec{Q}$ are the actual stress values at every integration point of all the interfaces in the model. Eqn. (4) guaranties that the yield functions, vector $\vec{\varphi}$, are not violated and eqn. (5) ensures that plastic flow implies energy dissipation. Finally, eqn. (6) guaranties that plastic flow cannot occur unless the stresses have reached the yield surface. Figure 1 shows a graphical representation of the static variables at two adjacent blocks, named $i$ and $j$, and the common quadrilateral interface, $k$. Details about these vectors, matrices and functions for rigid block, three-dimensional models have been given elsewhere [4]. In this paper, modifications are made with the aim to change the generalised stresses approach to the integration points approach.

$$
\begin{aligned}
\vec{N}_{0} \delta \vec{\lambda}-\vec{C} \delta \vec{u} & =\overrightarrow{0} \\
\vec{F}_{v}^{T} \cdot \delta \vec{u}-1 & =0 \\
\vec{F}_{c}+\alpha \vec{F}_{v}-\vec{C}^{T} \vec{Q} & =\overrightarrow{0} \\
\vec{\varphi} & \leq \overrightarrow{0} \\
\delta \vec{\lambda} & \geq \overrightarrow{0} \\
\vec{\varphi}^{T} \cdot \delta \vec{\lambda} & =0
\end{aligned}
$$



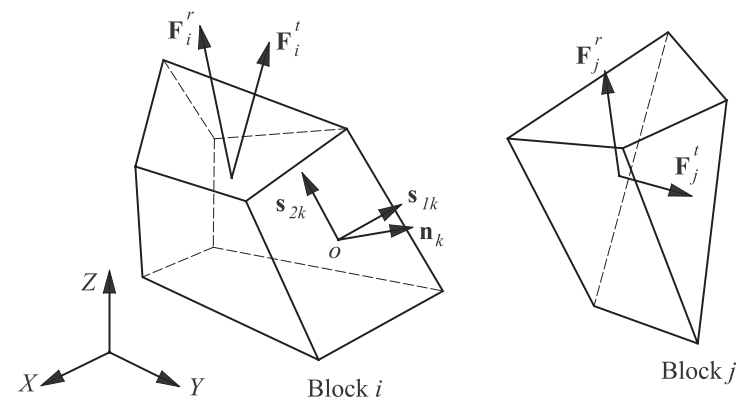

Figure 1: Static variables at an interface and adjacent blocks.

\section{Numerical integration approach}

Consider a quadrilateral shaped interface, $k$, illustrated in Figure 1. This interface lies between two infinitely strong blocks, $i$ and $j$. The cohesion-less Coulomb's law governs the interface failure. The interface is supposed to represent a dry masonry joint, therefore, it has no tensile strength. The origin of the local coordinate system is located at the centroid of the interface, $o$. This coordinate system has axes $x_{1}$ and $x_{2}$ on the interface plane, and $x_{n}$ in the normal direction, forming a right-handed system. The unitary vectors along the coordinate axes $x_{1}, x_{2}$ and $x_{n}$ are $\vec{s}_{1}, \vec{s}_{2}$ and $\vec{n}$, respectively, Figure 1 . The stress vector $\vec{\sigma}$ at a point has components $\tau_{1}, \tau_{2}$ and $\sigma$ along the axes $x_{1}, x_{2}$ and $x_{n}$, respectively. The normal component, $\sigma$, is positive in tension.

The resultant stresses over the interface are the shear forces, $V_{1}$ and $V_{2}$, along the $x_{1}$ and $x_{2}$ axes, respectively; the normal force, $N$; the bending moments, $M_{1}$ and $M_{2}$, along the $x_{1}$ and $x_{2}$ axes, respectively and the torsion moment, $T$. Under a particular stress distribution, eqns. (7-12) give the values for the stress resultants. Here, $\mathrm{d} A$ is the differential of area. The second right hand sides of eqns. (7-12) are the numerical integration approximations for the integrals in the first right hand sides. Here, $\xi$ represents the weights of the quadrature, $n_{p}$ is the number of integration points, assumed equal in both interface in-plane directions, $\|J\|$ is the determinant of the Jacobian of the transformation between the interface coordinate system and the quadrature coordinate system [8]. Since the interfaces are supposed to be quadrilaterals, this quantity is a constant and can be placed out of the summation. In the second right hand sides, the variables are evaluated only at the integration points, according to the indexes $l$ and $m$.

$$
\begin{array}{lll}
V_{1}= & \int_{\mathcal{S}} \tau_{1} \mathrm{~d} A & \doteq\|J\| \sum_{l=1}^{n_{p}} \sum_{m=1}^{n_{p}} \tau_{1 l m} \xi_{l} \xi_{m} \\
V_{2}= & \int_{\mathcal{S}} \tau_{2} \mathrm{~d} A & \doteq\|J\| \sum_{l=1}^{n_{p}} \sum_{m=1}^{n_{p}} \tau_{2 l m} \xi_{l} \xi_{m}
\end{array}
$$




$$
\begin{aligned}
& N=\quad \int_{\mathcal{S}} \sigma \mathrm{d} A \quad \doteq\|J\| \sum_{l=1}^{n_{p}} \sum_{m=1}^{n_{p}} \sigma_{l m} \xi_{l} \xi_{m} \\
& M_{1}=\quad \int_{\mathcal{S}} \sigma x_{2} \mathrm{~d} A \quad \doteq\|J\| \sum_{l=1}^{n_{p}} \sum_{m=1}^{n_{p}} \sigma_{l m} x_{2 m} \xi_{l} \xi_{m} \\
& M_{2}=\quad \int_{\mathcal{S}}-\sigma x_{1} \mathrm{~d} A \quad \doteq\|J\| \sum_{l=1}^{n_{p}} \sum_{m=1}^{n_{p}}-\sigma_{l m} x_{1 l} \xi_{l} \xi_{m} \\
& T=\int_{\mathcal{S}}\left(-\tau_{1} x_{2}+\tau_{2} x_{1}\right) \mathrm{d} A \doteq\|J\| \sum_{l=1}^{n_{p}} \sum_{m=1}^{n_{p}}\left(-\tau_{1 l m} x_{2 m}+\tau_{2 l m} x_{1 l}\right) \xi_{l} \xi_{m}
\end{aligned}
$$

In the approach proposed in this paper, it is not necessary to calculate the stress resultants over the interfaces, but it can be done by means of eqns. (7-12). The yield function characterisation is performed by limiting the normal and shear stresses at each integration point. The normal stress limits are: from bellow, the effective compressive stress and from top the zero value due to the no-tension hypothesis. Therefore, eqns. (13) and (14), represent the yield functions for the normal stress. Here, $f_{c e f}$ is the effective compressive stress, which takes into account the effect of transverse cracking and the fact that masonry presents quasi-brittle failure, while the model features perfect plastic behaviour. The shear stresses are limited by the cohesionless Coulomb criterion, as already mentioned. This criterion conducts to a quadratic function as illustrated in Figure 2 by the circle of radius $-\mu \sigma$, where $\mu$ is the friction coefficient. Nevertheless, in order to simplify the solution to the mathematical programming problem, a piecewise linear approximation is proposed and illustrated also in Figure 2 by an inscribed octagon. The resulting yield functions are expressed by eqns. (15) and (16). It is observed that, due to the absolute value operands, these two expressions represent the eight linear functions illustrated in Figure 2.

$$
\begin{array}{rlrl}
\varphi_{c} & \equiv & -\sigma-f_{c e f} & \leq 0 \\
\varphi_{t} & \equiv & \sigma & \leq 0 \\
\varphi_{s 1-s 4} & \equiv\left|\tau_{1}\right|+(\sqrt{2}-1)\left|\tau_{2}\right|+\mu \sigma \leq 0 \\
\varphi_{s 5-s 8} & \equiv(\sqrt{2}-1)\left|\tau_{1}\right|+\left|\tau_{2}\right|+\mu \sigma \leq 0
\end{array}
$$

There are, therefore, ten linear yield functions for each integration point. The yield functions for all the integration points at every interface in a model can be cast in matrix form, in such a way that eqn. (4) takes the form of eqn. (17). Here, $\vec{N}$ is the matrix resulting from the assemblage of all the yield functions in the model.

$$
\vec{\varphi} \equiv \vec{N} \vec{Q} \leq \overrightarrow{0}
$$

There are three generalised strain or relative displacement rate components at each integration point: $\delta s_{1}, \delta s_{2}$ and $\delta n$, parallel to the local coordinate directions 


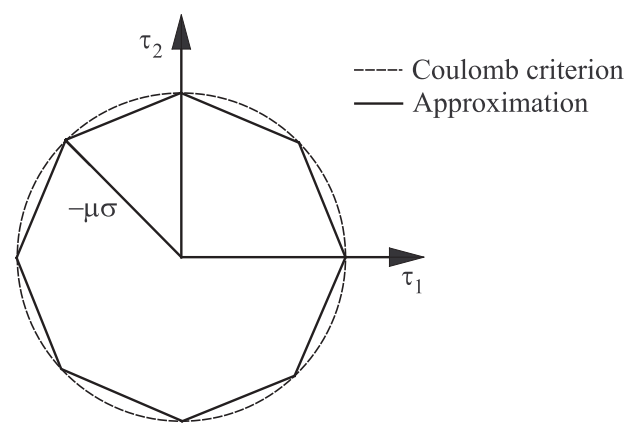

Figure 2: Shear yield function.

Table 1: Flow directions for an integration point failure.

\begin{tabular}{cccc}
\hline Flow multiplier & $\delta s_{1}$ & $\delta s_{2}$ & $\delta n$ \\
\hline$\delta \lambda_{c}$ & 0 & 0 & -1 \\
$\delta \lambda_{t}$ & 0 & 0 & 1 \\
$\delta \lambda_{s 1-4}$ & $\tau_{1} /\left|\tau_{1}\right|$ & $(\sqrt{2}-1) \tau_{2} /\left|\tau_{2}\right|$ & 0 \\
$\delta \lambda_{s 5-8}$ & $(\sqrt{2}-1) \tau_{1} /\left|\tau_{1}\right|$ & $\tau_{2} /\left|\tau_{2}\right|$ & 0 \\
\hline
\end{tabular}

$x_{1}, x_{2}$ and $x_{n}$, respectively. The flow directions for the yield modes are in Table 1. For the compression and tension yield modes the flow consists on a normal relative displacement rate in the negative and positive directions of the $x_{n}$ axis, respectively. The flow directions for the sliding failure modes are obtained from the normality rule but neglecting the relative displacement rate along the $x_{n}$ axis, due to the hypothesis of zero dilatancy. The rows of Table 1 are used to assemble the columns of matrix $\vec{N}_{0}$, in eqn. (18), which gives the generalised strains resulting from the flow rule condition. Here the vector $\delta \vec{q}$ gathers the generalised strains for all the integration points in a model.

$$
\delta \vec{q}=\vec{N}_{0} \delta \vec{\lambda}
$$

The displacement rates for a generic block $i$ are the translation displacement rates at the block centroid $\delta \vec{u}_{i}^{t}$ and the angular displacement rates $\delta \vec{u}_{i}^{r}$. These vectors are referred to the global coordinates system. Eqn. (19) gives the generalised strains at the integration point $p$ of the interface $k$ in terms of the displacement rates at the blocks $i$ and $j$; see Figure 1 . Here, $\vec{T}_{k}^{g}$ is the matrix that transforms a vector from the global coordinates system to the local interface $k$ system; $\vec{c}_{p k}, \vec{c}_{i}$ and $\vec{c}_{j}$ are the position vectors of the point $p$, and the centroids of blocks $i$ and $j$, respectively. Eqn. (19) can be used to assemble the compatibility set of equations 
of a model in the form of eqn. (20). It is evident that eqn. (1) follows directly from eqns. (18) and (20).

$$
\begin{aligned}
\delta \vec{q}_{p k} & =\vec{T}_{k}^{g}\left(\delta \vec{u}_{j}^{t}-\delta \vec{u}_{i}^{t}+\delta \vec{u}_{j}^{r} \wedge\left(\vec{c}_{p k}-\vec{c}_{j}\right)-\delta \vec{u}_{i}^{r} \wedge\left(\vec{c}_{p k}-\vec{c}_{i}\right)\right) \\
\delta \vec{q} & =\vec{C} \delta \vec{u}
\end{aligned}
$$

\section{Torsion failure on frictional interfaces}

Orduña and Lourenço [4] studied the torsion failure on rectangular frictional interfaces. In that work, the assumptions were made that dilatancy coefficient was zero and that the normal stresses over the interface have uniform distributions either over all or part of the interface area. With these assumptions, yield functions and flow rules were presented in terms of the stress resultants over the interface. Nevertheless, with the proposal presented in this work, where the normal stresses at each integration point of an interface are independent each other, there are no guarantee that uniform or even normal stresses distributions are obtained over an interface. This section presents a discussion about the influence of the normal stresses distribution over the interface on its torsion moment strength.

Consider a rectangular interface with dimensions $2 l_{1}$ and $2 l_{2}$ parallel to the local axes $x_{1}$ and $x_{2}$, respectively, as shown in Figure 3. This interface, placed between two infinitely rigid bodies, is subjected to a constant normal force in compression, $N$ (negative), at the interface centroid, and fails under the action of a torsion moment, $T$. The friction coefficient is $\mu$ and an infinite compressive strength is assumed by now.

At incipient failure, the sliding displacement rates are zero at the centre of twisting and vary linearly with the distance from this point. If associated plasticity is assumed (dilatancy coefficient equals friction coefficient), the normal displacement rates would be proportional to the sliding displacement rates. The limit

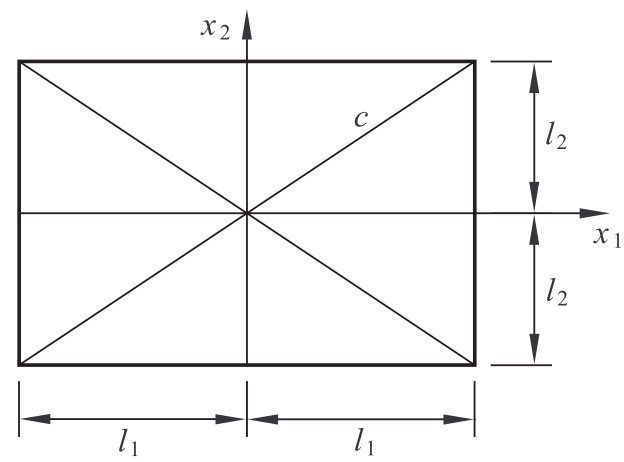

Figure 3: Rectangular joint. 
analysis solution indicates that the centre of twisting is located at the interface centroid, therefore, the maximum sliding and normal displacement rates are at the interface corners. As the blocks are rigid, separation will occur at the inner points, and contact will be possible only at the interface corners. Under this conditions, it is straightforward to show that eqn. (21) proportionates the value of the failure torsion moment, where $c$ is the distance from the interface centroid to any corner; see eqn. (22) and Figure 3. Michalowski and Gawecki [9] arrived to an analogous conclusion for a circular interface where $c$ is replaced by the circle radius in eqn. (21). It is possible to arrive to the same result always that the dilatancy coefficient is positive, even if it does not equal the friction coefficient.

$$
\begin{aligned}
T & =c \mu(-N) \\
c & =\sqrt{l_{1}^{2}+l_{2}^{2}}
\end{aligned}
$$

If the dilatancy coefficient is negative, the normal displacement rates are again proportional to the sliding displacement rates. Nevertheless, no separation but penetration exists between blocks, contact is only possible at the very interface centroid and, therefore, the torsion moment strength is zero (contact on a single point).

If the dilatancy coefficient is zero, contact points or areas are not determined by the failure mechanism and neither, the normal stress distribution. This means that for every possible normal stress distribution, there exists a torsion moment strength. It is evident that the values of these strengths lie between zero and that given by eqn. (21). The most fiscally meaningful case consists on a uniform normal stress distribution over the interface. For this case, eqn. (23) gives the torsion moment strength, where the torsion constant $c_{\mathrm{T}}$ is given by eqn. (24) [4]. It is observed that eqns. (21) and (23) are the same except for the torsion constant definition.

$$
\begin{aligned}
T & =c_{\mathrm{T}} \mu(-N) \\
c_{\mathrm{T}} & =\frac{1}{3}\left[c+\frac{l_{1}^{2}}{2 l_{2}} \ln \left(\frac{l_{2}+c}{l_{1}}\right)+\frac{l_{2}^{2}}{2 l_{1}} \ln \left(\frac{l_{1}+c}{l_{2}}\right)\right]
\end{aligned}
$$

The $c_{T} / c$ ratio can be calculated for the whole range of interface aspect ratios, and the observation is made that it has a small variation between 0.50 and 0.54 . Therefore, a first but marginal conclusion is that the torsion constant can be approximated as half the distance from the centroid to the corner. A more important observation is that, for zero dilatancy, the torsion strength of the interface can take values between zero and that given by eqn. (21), but the strength for a uniform normal stress distribution is about half the way between the former values. Therefore, it is of fundamental importance, in limit analysis problems with zero dilatancy, to obtain solutions with even normal stress distributions over the interfaces.

Orduña and Lourenço [4, 10] showed that for this type of problem it is also important to take into account the loading history. Here, a solution procedure, akin 
Table 2: Calculated ultimate load factors for infinite compressive strength.

\begin{tabular}{lc}
\hline Procedure & Ultimate load factor \\
\hline Theoretically minimum & 0.427 \\
Theoretically maximum & 0.553 \\
FEM & 0.479 \\
Load-path following & 0.465 \\
\hline
\end{tabular}

to the load-path following one is used, which agrees with the integration points approach. The main idea behind this procedure is to keep the normal stresses distribution as uniform as possible, while the variable loads are applied by small increments.

\section{Validation}

The validation of this type of three-dimensional models is not an easy task due to the lack of experimental or analytical results to compare with. Therefore, the example presented here is a comparisons against a finite element (FEM) non-linear model analysed with the package DIANA [11].

Figure 4(a) shows the model of a masonry hollow pile. The pile is built of dry masonry blocks with dimensions $0.2 \times 0.2 \times 0.4 \mathrm{~m}$. The pile dimensions are $0.6 \times 0.8 \times 1.2 \mathrm{~m}$. The material volumetric weight is $20 \mathrm{kN} / \mathrm{m}^{3}$ and the friction coefficient is 0.7 . The permanent loads are the self-weight of the blocks. The variable loads are proportional to the blocks weight, but horizontally applied in the direction of the larger base side ( $X$ direction). The compressive effective stress, according to the load-path following procedure, is steadily increasing.

Figure 4(b) shows the failure mechanism obtained by the FEM analysis. The same failure mechanism is obtained by limit analysis and Figures 4(c), (d) show it from two different viewpoints for a better understanding. A range of ultimate load factors is possible for this mechanism and for infinite compressive strength. It is noted that the ultimate load factor is defined as the ratio between the variable loads causing failure on the structure, and their nominal values, in this case numerically equal to the self-weight of the blocks. Table 2 presents the ultimate load factor obtained from different approaches. If the reaction on the overturning blocks set is concentrated on the interface $A$ only, Figure 4(d), with zero stresses at interface $B$, the ultimate load factor would be 0.427 , the minimum possible for this mechanism. If there are non-zero normal and shear contact forces on interface $B$, the last one opposing to the upper block overturning, the maximum possible ultimate load factor for the mechanism shown equals 0.553 . The ultimate load factor calculated with the load-path following procedure is $3 \%$ lower than the FEM value. It is possible to verify that the load-path following procedure agrees very well with the FEM results. 


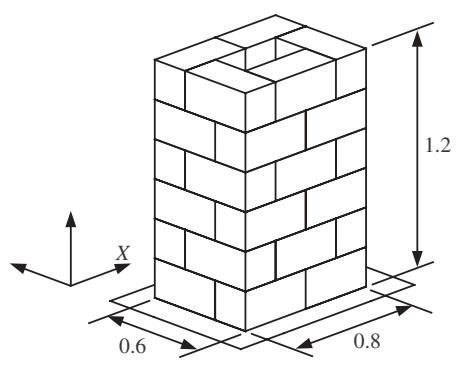

(a)

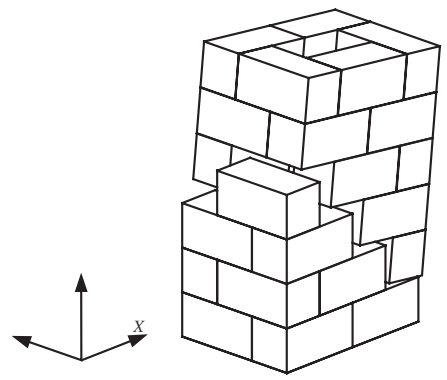

(c)

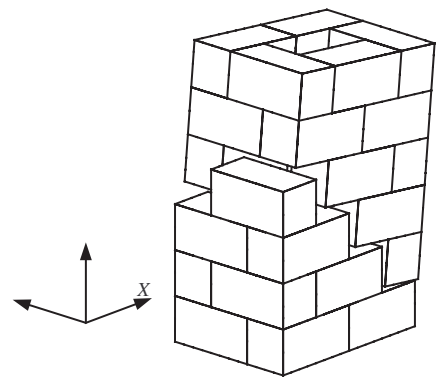

(b)

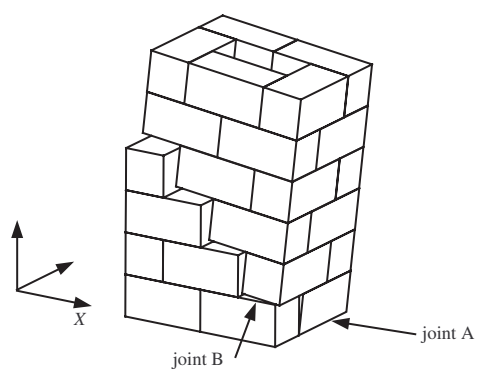

(d)

Figure 4: Masonry pile; (a) model; failure mechanisms for infinite compressive strength: (b) FEM failure mechanism; (c) and (d) different views of the limit analysis failure mechanism.

It is interesting to note that the condition of zero stresses at interface $B$ is only possible for an infinite compressive strength. Under limited compressive stresses, the hinge on interface $A$ forms slightly inwards and interface $B$ must be in contact and must transmit normal and shear forces.

\section{Conclusions}

A limit analysis formulation using numerical integration techniques at the interfaces has been proposed for rigid block assemblages. The importance of even normal stress distributions over the interfaces was demonstrated. Comparisons, not presented here due to space limitations, show that good agreement is obtained, at interface level, between the integration points approach and the yield functions obtained by constant normal stress distributions over regular shaped parts of the 
interface. The presented example shows also very good agreement compared with the non-linear FEM results. It must be recognised that the computing time for the limit analysis approach and for this large model is not attractive compared with the FEM process. Nevertheless, it is expected that, optimising the numerical tasks for the limit analysis approach, advantage can be taken from the simplified nature of this formulation and the computing times can be significantly reduced.

\section{Acknowledgment}

This work was supported by project PROMEP/103.5/04/1322 funded by the Secretary of Public Education (SEP) of Mexico.

\section{References}

[1] Begg, D. \& Fishwick, R., Numerical analysis of rigid block structures including sliding. Computer Methods in Structural Masonry 3, eds. J. Middleton \& G. Pande, Portugal, pp. 177-183, 1995.

[2] Baggio, C. \& Trovalusci, P., Limit analysis for no-tension and frictional three-dimensional discrete systems. Mech Struct Mach, 26(3), pp. 287-304, 1998.

[3] Ferris, M. \& Tin-Loi, F., Limit analysis of frictional block assemblies as a mathematical program with complementarity constraints. Int J Mech Sci, 43, pp. 209-224, 2001.

[4] Orduña, A. \& Lourenço, P., Three-dimensional limit analysis of rigid blocks assemblages. Part I: Torsion failure on frictional interfaces and limit analysis formulation. Int J Solids Structures, 2005. Accepted for publication.

[5] Livesley, R., Limit analysis of structures formed from rigid blocks. Int J Num Meth Engrg, 12, pp. 1853-1871, 1978.

[6] Livesley, R.K., A computational model for the limit analysis of threedimensional masonry structures. Meccanica, 27, pp. 161-172, 1992.

[7] Orduña, A. \& Lourenço, P., Cap model for limit analysis and strengthening of masonry structures. J Struct Eng, 129(10), pp. 1367-1375, 2003.

[8] Bathe, K.J., Finite Element Procedures in Engineering Analysis. PrenticeHall, 1982.

[9] Michalowski, R.L. \& Gawecki, A., Limit torque for a frictional joint. Mech Struct Mach, 24(4), pp. 499-512, 1996.

[10] Orduña, A. \& Lourenço, P.B., Three-dimensional limit analysis of rigid blocks assemblages. Part II: Load-path following solution procedure and validation. Int J Solids Structures, 2005. Accepted for publication.

[11] TNO Building and Construction Research, Delft, The Netherlands, DIANA User's Manual Release 7.2, 1999. 